\title{
An experimental model of chronic rhinosinusitis in rabbits without bacterial inoculation ${ }^{1}$
}

\author{
Raphaella de Oliveira Migliavacca ${ }^{\mathrm{I}}$, Otávio Bejzman Piltcher ${ }^{\mathrm{II}}$, Lúcia Maria Kliemann ${ }^{\mathrm{III}}$, Marcelle Reesink Cerski' ${ }^{\mathrm{II}}$, Fabíola \\ Meyer $^{\text {IV }}$, Paula de Oliveira Oppermannv, Geraldo Machado FilhovI, Suzie Hyeona Kang ${ }^{\text {VII }, \text { Sady Selaimen da Costa }}{ }^{\text {VIII }}$
}

\author{
DOI: http://dx.doi.org/10.1590/S0102-86502014000500005
}

${ }^{\text {I}}$ Fellow Master degree, Postgraduate Program in Medicine Surgical Sciences, Rio Grande do Sul Federal University (UFRGS), Brazil. Conception and design of the study, acquisition and interpretation of data, surgical procedures, manuscript preparation, English language.

IIPhD, Full Professor, Department of Otolaryngology, School of Medicine, UFRGS, Brazil. Conception and design of the study; surgical procedures; acquisition, interpretation and analysis of data, critical revision.

IIIPhD, Full Professor, Department of Pathology, School of Medicine, UFRGS, Brazil. Histopathological examinations, acquisition of data.

${ }^{\text {IV }}$ MSc, Veterinarian, Experimental Research Center (CPE), Porto Alegre Clinics Hospital, Brazil. Anesthesia, technical procedures.

${ }^{\vee} \mathrm{MD}$, Resident, Division of Otolaryngology, Porto Alegre Clinics Hospital, Brazil. Acquisition of data, manuscript preparation.

${ }^{\mathrm{VI}}$ Graduate student, School of Medicine, UFRGS, Brazil. Acquisition of data, technical procedures.

${ }^{\mathrm{VII}}$ Fellow PhD degree, Postgraduate Program in Pneumology Sciences, UFRGS, Brazil. Surgical procedures, acquisition of data.

VIIIPhD, Full Professor, Department of Otolaryngology, UFRGS, Brazil. Conception of the study, critical revision.

\begin{abstract}
PURPOSE: Evaluate and compare two different experimental techniques of maxillary sinus ostium occlusion using N-butyl cyanoacrylate in developing chronic histological findings without the inoculation of pathogenic bacteria among rabbits.

METHODS: In a randomized study, sixteen New Zealand rabbits were assigned for occlusion of the right maxillary sinus through a transmaxillary approach or through the roof of the nasal cavity. The contralateral sinus served as a control. After 12 weeks, the animals were sacrificed for blinded histopathological analysis of the maxillary sinus mucosa.

RESULTS: Histopathological changes consistent with CRS were found in eight (100\%) of the maxillary sinuses approached transmaxillary and three of those through the roof of the nasal cavity (37.5\%), p 0.008 and 0.250 , respectively, comparing with the control side. Chronic mucosal changes were significantly better induced using the transmaxillary approach (p 0.026).

CONCLUSION: It is possible to induce a model of chronic sinusitis among rabbits with transmaxillary sinus occlusion without bacterial inoculation. This model can be replicated for future cellular studies.
\end{abstract}

Key words: Sinusitis. Disease Models, Animal. Models, Animal. Maxillary Sinusitis. Rabbits. 


\section{Introduction}

According to U.S. statistics, chronic rhinosinusitis (CRS) affects approximately 35 million American individuals and generates a cost of $\$ 6$ billion annually ${ }^{1,2}$. In Brazil, despite being recognized as one of the most common disorders of the upper airways, there is a lack of well-designed epidemiological studies ${ }^{3}$.

While we know that CRS has a high prevalence, there is still much to understand about the pathogenesis of rhinosinusitis and its categorization, in order to reduce inappropriate practices. Apparently, the obstruction of the ostiomeatal complex is one of the initiating events that leads to hypoxia, anaerobic metabolism, and an acidic environment that inhibits the body's local immune mechanisms and promotes colonization by pathogenic bacteria. Histologically the disease is established when persistent inflammatory changes are seen, with edema and desquamation of the ciliated respiratory epithelium, squamous metaplasia, fibrosis, globet cell hyperplasia, subepithelial thickening, polyp formation and disruption of the mucociliary transport mechanism ${ }^{1}$. The triggers between these steps are still obscures.

In this context, experimental models have an important role and the rabbit model is the most widely used. The maxillary sinus of rabbits resembles that of humans in many ways: a welldefined ostium, a wide and accessible cavity, and a mucociliary system with comparable features ${ }^{4}$. Most studies that focus on experimental models of rhinosinusitis associate maxillary sinus ostium occlusion with the inoculation of bacteria. Moreover, most studies have a short follow-up period, usually days, not reaching the twelve weeks period considered for diagnostic of a chonic process.

The aim of the present study is to produce a consistent and reproducible experimental model among rabbits of chronic maxillary rhinosinusitis, without the inoculation of pathogenic bacteria, secondary to sinus ostium obstruction. Secondarily, we compared the inflammatory findings resulting from the two techniques of maxillary sinus ostium occlusion with N-butyl cyanoacrylate: transmaxillary and through the roof of the nasal cavity.

\section{Methods}

Research approved by the Ethics Committee on Animal Use of UFRGS ( $n^{\circ}$ 110292). The use of laboratory animals followed the Council for International Organization of Medical Sciences (CIOMS) ethical code for animal experimentation. Sixteen male New Zealand rabbits (Oryctolagus cuniculus), Pasteurella-free, aged around 120 days and weighing 2700-
$3500 \mathrm{~g}$, were allocated to the study. The rabbits arrived at the Unit at least three days before surgery for a period of adaptation, when the first intramuscular injection of oxytetracycline $(20 \mathrm{mg} /$ $\mathrm{kg}$ ) was applied; in an interval of three days, a second dose of antibiotic prophylaxis was administered. This drug was given due to the high prevalence of Pasteurella multicida and the possibility of subclinical infection. Sample size was determined through literature review and pilot studies, in which a success rate of sinus occlusion and induction of rhinosinusitis was estimated as $80 \%$ in each group. Considering a statistical power of $80 \%$, seven rabbits in each arm were calculated and one rabbit to each group was added.

Exclusion criteria were the following: (1) identification of pus in the maxillary sinus during surgery; (2) presence of rhinosinusitis (sinus mucosal hyperplasia with an infiltrate of inflammatory cells) in the transoperative biopsy; (3) death of the animal.

All procedures were performed by the same surgeon. After premedication (pethidine $5 \mathrm{mg} / \mathrm{kg}$, midazolam $1 \mathrm{mg} / \mathrm{kg}$, and ketamine $15 \mathrm{mg} / \mathrm{kg}$ ), inhalation anesthesia (isoflurane) and intubation, a sealed envelope prepared by a blinded research assistant was opened to verify the procedure to be performed. Rabbits were randomly assigned to the following groups:

Transmaxillary group (TM group): occlusion of the right maxillary sinus ostium, approached through the maxillary sinus, under direct visualization. Following aseptic procedures with iodine and infiltration of bupivacaine $1.5 \mathrm{mg} /$ $\mathrm{kg}$ and lidocaine $2 \mathrm{mg} / \mathrm{kg}$ (Figure 1A), the maxillary sinus was approached through a vertical incision without reaching the periosteum. Then, a periosteal flap was elevated and fixed for exposure of the maxillary bone (Figure 1B). A small opening of $4-5 \mathrm{~mm}$ was drilled on the upper maxillary sinus (Figure 1C), preserving the underlying mucosa. Before opening the maxillary sinus mucosa, a 3-mL syringe filled with air was used to test for movement of the mucosa and hence ostial permeability (Figure 1D). Then, the mucosal layer was incised with a scalpel, opening the right maxillary sinus and allowing the visualization of the mucosal surface, checking for the presence of possible secretions and the identification of the ostium (Figure 2). Next, the ostium was occluded using subcutaneous tissue from the same animal ( $5 \times 3 \mathrm{~mm}$ ) and two drops of N-butyl cyanoacrylate (Vetbond $^{\circledR}$, 3M, St. Paul, MN, USA). The sinus mucosa was then biopsied. The periosteal flap was repositioned and the air syringe test was repeated to observe for any movement, which would indicate the need to reposition the material for effective occlusion. Finally, the wound was closed in layers. 


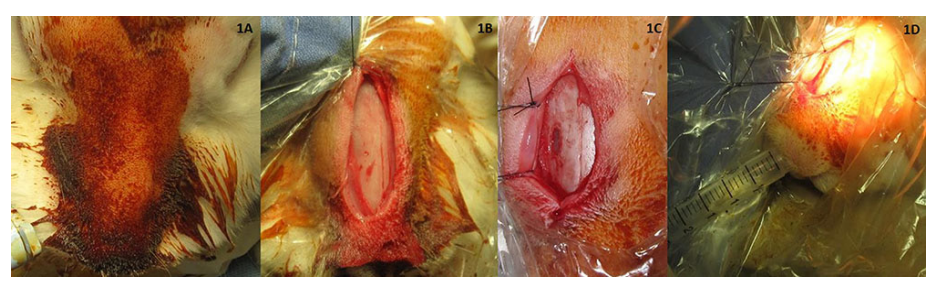

FIGURE 1 - Sequence of procedure performed in all cases: antissepsis (A); access and exposure of the maxillary bone, with visualization of septum, nasal cavities and maxillary sinus by transparency (B); fenestration of 4-5 $\mathrm{mm}$ in the upper half of maxillary sinus without disruption of the underlying mucosa (C); the test of ostium patency (D).

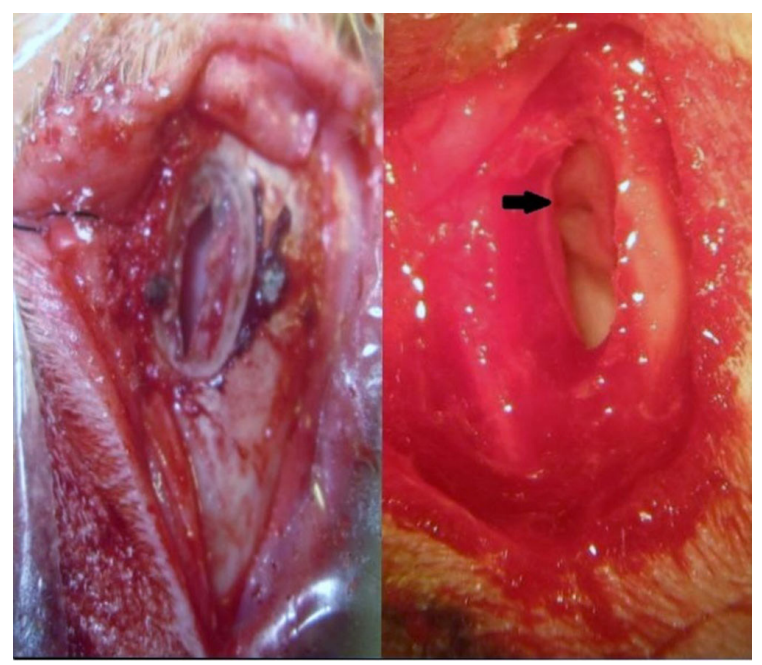

FIGURE 2 - The aspect of the lumen and mucosa of the maxillary sinus and identification of the ostium (arrow).

Roof of the nasal cavity group (RNC group): occlusion of the right middle meatus and visualization through the roof of the nasal cavity. The initial surgical approach was performed similarly as described for the TM Group. However, the drilling was between the nasal septum and the nasal cavity. The lower and inner corner of the ipsilateral eye was established as the upper reference point (3 medial $\mathrm{cm}$ ) (Figure 3). After drilling, the endoturbinate (similar to middle turbinates in humans) was exposed and placed medially. The region of the ostium of the maxillary sinus was identified and occluded with subcutaneous tissue and two drops of N-butyl cyanoacrylate. The air syringe test was performed after repositioning of the flap to check for any movement of the right maxillary sinus mucosa. Then the sinus was opened with a scalpel for biopsy, followed by closure in layers.

No procedure was performed on the left side in both groups at the time.

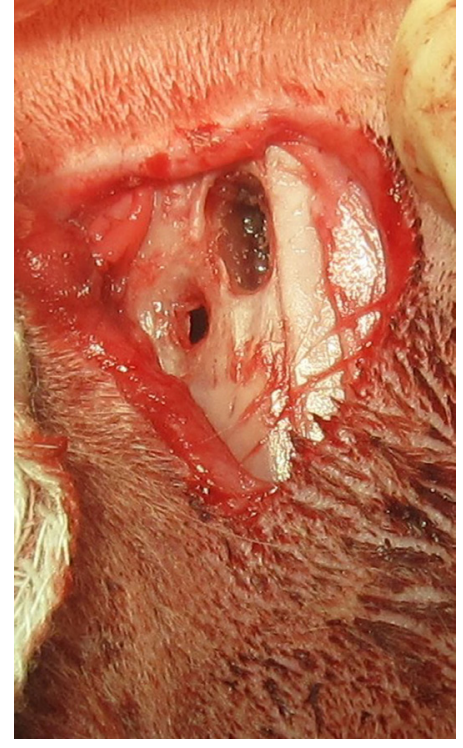

FIGURE 3 - Approach through roof of the nasal cavity.

After 12 weeks under veterinary care, animals were sedated with midazolam $(1 \mathrm{mg} / \mathrm{kg})$ and ketamine $(15 \mathrm{mg} / \mathrm{kg})$, followed by the administration of an overdose of propofol (15 $\mathrm{mg} / \mathrm{kg}$ ). Finally, an injection of $5 \mathrm{ml}$ of potassium chloride $10 \%$ was used to cause cardiac arrest. Immediately after the death of each animal, material was collected directly from each maxillary sinus through the osteotomy and cultured in liquid culture media (BacT/ALERT ${ }^{\circledR}$, Biomerieux, Durham, NC, USA).

A coronal cut was made through the paranasal sinuses to check for the presence of macroscopic material occluding the right maxillary sinus ostium. For light microscopy (Olympus BX41; Olympus Optical Co., Tokyo, Japan) the decalcificated samples (10\% nitric acid for 24 hours) were fixed in $10 \%$ formaldehyde solution and embedded in paraffin. Five-micrometer sections were cut from paraffin blocks and stained with hematoxylin and eosin. Stained randomly numbered slides were observed by two pathologists blinded to the intervention.

Statistical analysis was performed using SPSS program (PASW Statistics 17.0; SPSS, Inc., Chicago, IL). Categorical variables were expressed as proportions or median and interquartile ranges (ordinal variables). McNemar's exact test was used to test the difference between proportions, except for variables with more than two levels, when the Wilcoxon test was used. Fisher's exact test was used to compare differences between surgical techniques in dichotomous categorical variables and the Mann-Whitney test for ordinal variables. Both Kappa index and percentage of agreement were used to verify interobserver agreement for histopathological variables. 


\section{Results}

None of the animals presented criteria to be excluded from the study.

Through the coronal cut that it was found the presence of macroscopic material occluding the right maxillary sinus ostium in eight rabbits in the TM group, with visualization of frank purulent drainage in the right maxillary sinuses of six of eight rabbits (Figure 4). In the RCN group, presence of occluding material, accompanied by purulent drainage, was evident in the right maxillary sinus ostium of five rabbits; partial or questionable occlusion was present in two and one animal had no signs of secretion in the maxillary sinus ostium.

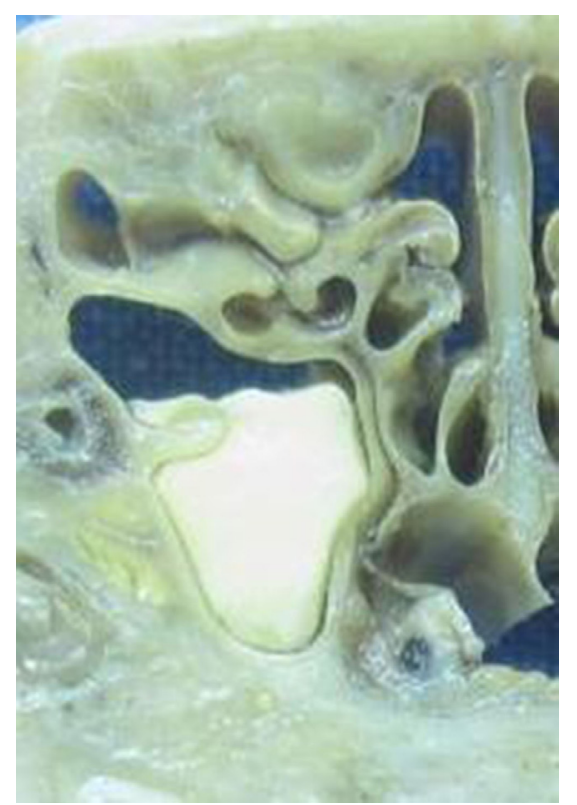

FIGURE 4 - Intense purulent discharge inside the right maxillary sinus in post-mortem macroscopic study of an animal of transmaxillary approach.

\section{Histological changes compatible with CRS (defined} as hyperplasia of sinus mucosa with dense infiltrate of chronic inflammatory cells $)^{2}$ were found in the mucosa of the right maxillary sinus of eight rabbits $(100 \%)$ that underwent occlusion through the transmaxillary approach (Figure 5A), with statistical significance when compared to the control side (p 0.008). Three right maxillary sinuses (37.5\%) of rabbits submitted to occlusion with the RNC approach showed signs of CRS (Figure 5B). Control plates, representing the opposite side of the occlusion, showed normal (Figure 5C) or atrophic mucosa (Figure 5D), as well as absence of any findings consistent with CRS. Table 1 shows the histopathological findings for the two groups. Comparing the occlusion techniques (Table 2), the TM approach proved to be more consistent in causing chronic changes in the mucosa of the occluded maxillary sinuses ( $p$
0.026). The median Kappa agreement among pathologists was 0.67 for the occluded side and 0.74 for the control side. The median percentage of agreement among pathologists was $88 \%$.

Microbiological data of the maxillary sinus secretions collected at 12 postoperative weeks are shown in Table 3.

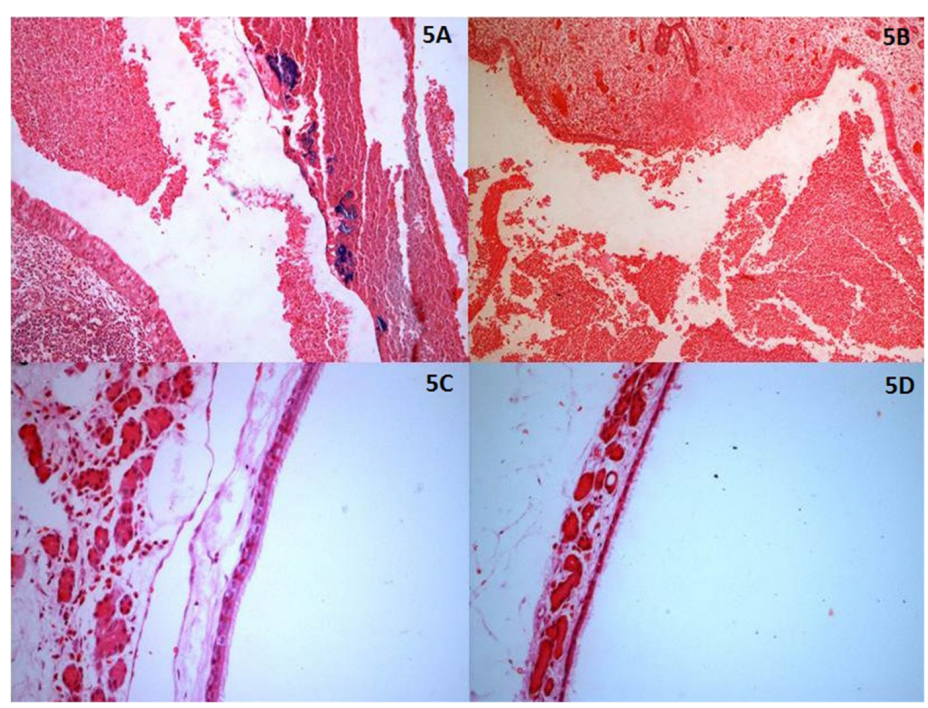

FIGURE 5 - Histopathologic analysis showing some findings: inflammatory infiltrate, exudate in the lumen and bacterial colonies in the right maxillary sinus of a transmaxillary case, x200 magnification (A); right maxillary sinus of an animals subjected to occlusion through the roof of the nasal cavity showing exudate in the lumen, inflammatory infiltrate and granulation tissue, x200 magnification (B); normal (C) and atrophic mucosa (D) in control side, x400 magnification.

TABLE 1 - Macroscopic and histopathologic data comparison between occluded side and control side (McNemar test for exact method except*)

\begin{tabular}{lcccccc}
\hline & \multicolumn{3}{c}{ Transmaxillary approach } & \multicolumn{3}{c}{$\begin{array}{c}\text { Roof of the nasal cavity } \\
\text { approach }\end{array}$} \\
\hline & $\begin{array}{c}\text { Occlusion } \\
\text { side }\end{array}$ & $\begin{array}{c}\text { Control } \\
\text { side }\end{array}$ & P & $\begin{array}{c}\text { Occlusion } \\
\text { side }\end{array}$ & $\begin{array}{c}\text { Control } \\
\text { side }\end{array}$ & $\mathrm{P}$ \\
\hline $\begin{array}{l}\text { Macroscopic } \\
\text { occlusion }\end{array}$ & $8 / 8$ & $0 / 8$ & 0.008 & $\begin{array}{c}5 / 8 \text { (total) } \\
2 / 8 \text { (semi) }\end{array}$ & $0 / 8$ & $0.45^{*}$ \\
\hline $\begin{array}{l}\text { Chronic } \\
\text { rhinosinusitis }\end{array}$ & $8 / 8$ & $0 / 8$ & 0.008 & $3 / 8$ & $0 / 8$ & 0.250 \\
\hline * For comparison of variables with more than two levels was used the Wilcoxon test
\end{tabular}


TABLE 2 - Data comparison between techniques in the right maxillary sinus (intervention) (Fisher's exact test)

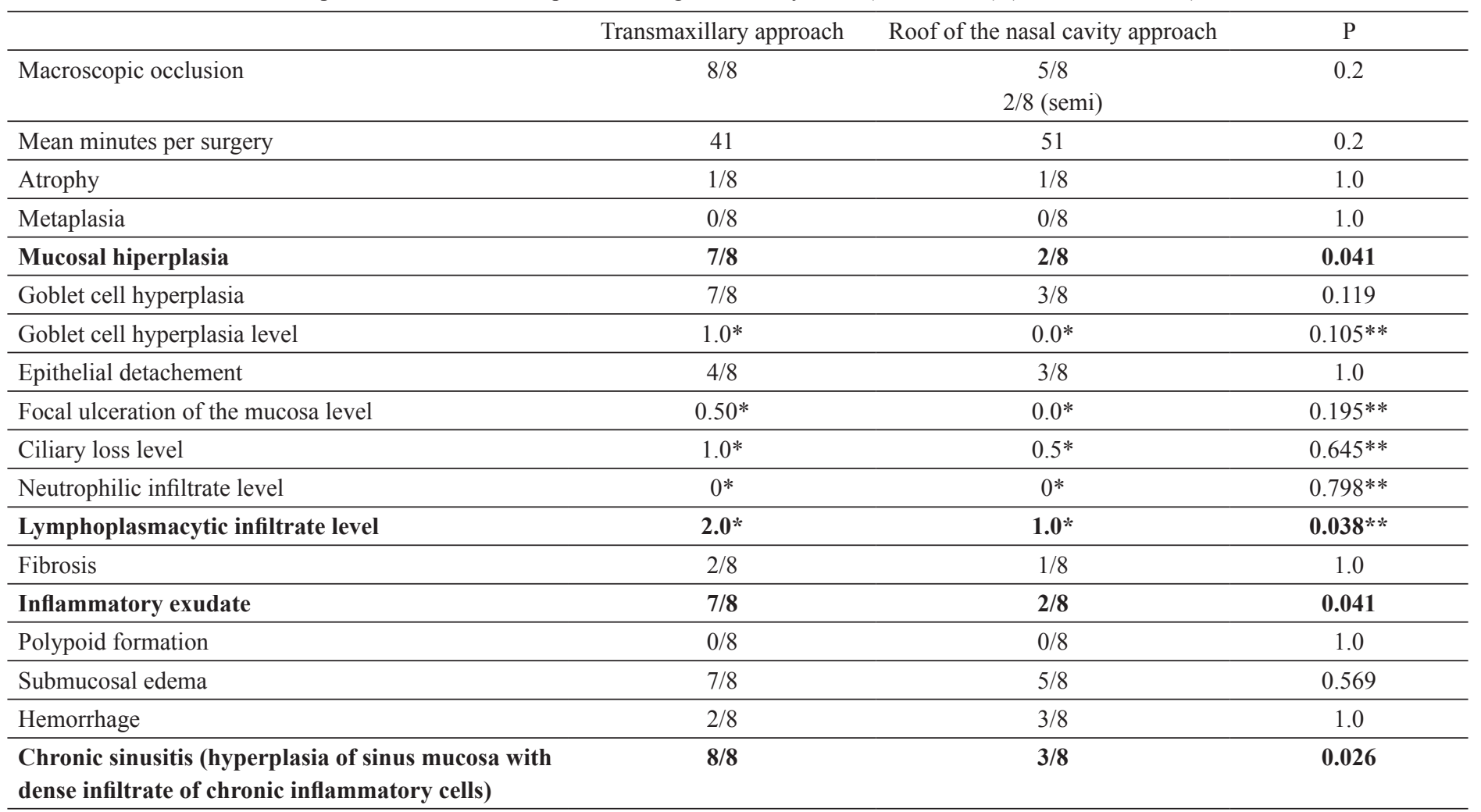

*The variables with more than two levels are presented as median

**For comparison of ordinal variables it was used the Mann-Whitney test

TABLE 3 - Microbiological data

\begin{tabular}{|c|c|c|c|}
\hline Rabbit & Approach & Occlusion side & Control side \\
\hline 01 & TM group & escherichia coli & gram negative bacillus \\
\hline 02 & TM group & gram negative bacillus & gram negative bacillus \\
\hline 04 & $\mathrm{RNC}$ group & gram negative bacillus & Negative \\
\hline 05 & TM group & Negative & Negative \\
\hline \multirow[t]{2}{*}{07} & RNC group & - staphylococcus sp. coagulase negative & gram negative bacillus \\
\hline & & - gram negative bacillus & \\
\hline 08 & TM group & gram negative bacillus & gram negative bacillus \\
\hline 09 & $\mathrm{RNC}$ group & enterobacter sp. & negative \\
\hline 10 & TM group & gram negative bacillus & gram negative bacillus \\
\hline 14 & TM group & gram negative bacillus & gram negative bacillus \\
\hline 15 & TM group & gram negative bacillus & gram negative bacillus \\
\hline 16 & $\mathrm{RNC}$ group & gram negative bacillus & negative \\
\hline
\end{tabular}

TM: Transmaxillary approach/RNC: Roof of the nasal cavity approach 


\section{Discussion}

The main goal of this study was to create a consistent and reproducible experimental model of CRS comparing two different techniques. The results showed clearly that this was possible in animals submitted to direct ostium obstruction through a transmaxillary approach. However theoretically more similar to human pathogenesis as the obstruction starts in the nose the technique through the roof of the nasal cavity did not show the same consistency in findings, with considerable heterogeneity in the macroscopic observations and histopathological features. This is an inherent difference that may not affect the conclusions drawn from the study, but it must be kept in mind in order to not extrapolate without strict criteria. Beyond question, the development of sinus mucosa with a cellular pattern consistent with CRS creates field that may help the advancing on cellular studies of this disease.

The utilization of rabbits in experimental sinus surgery is described since $1941^{5}$. Several examples of techniques to experimentally induce sinusitis have been proposed since then. Johansson et al. ${ }^{6}$ developed a classical study of acute sinusitis with a total of 69 rabbits divided into four groups (ostial obstruction with N-butyl cyanoacrylate and inoculation of bacteria; ostial obstruction without inoculation of bacteria; inoculation of bacteria without obstruction; and injection of N-butyl cyanoacrylate to investigate whether it could provoke chemical sinusitis). The experimental group assigned to the combined procedure developed acute rhinosinusitis. Administration of N-butyl cyanoacrylate did not induce histopathological findings suggestive of chemical sinusitis. According to the authors, ostial obstruction without bacterial inoculation was not sufficient to induce sinusitis in rabbits. Thereafter, other studies were published sequentially testing inoculation of different bacteria in the sinus of rabbits, with different follow-up from the induction of the rhinosinusitis until the animal sacrifice ${ }^{7-9}$. However, a Brazilian study ${ }^{10}$ which aimed to compare the development of acute rhinosinusitis with four different interventions (surgical sponge filling the nasal cavity; ostial obstruction with the instillation of cyanoacrylate into the infundibulum; percutaneous instillation of venous blood into the maxillary sinus; and percutaneous instillation of streptococcal and staphylococcal toxoid in the maxillary sinus) described the development of sinusitis in all groups, except with the instillation of peripheral blood. In the present study, we found that this technique, when used a transmaxillary approach, resulted in histopathological findings compatible with sinusitis in all maxillary sinuses, in agreement with the report discussed above, although the latter focused on a model of acute inflammatory disease.
Some authors are emphatic in criticizing the models of direct ostial obstruction, arguing that they were based on a sinusogenic model of rhinosinusitis, when the pathophysiological process was related to the nasal component. Thus, techniques to experimentally induce sinusitis with access through the nasal fossa have been described. Hinni et al. ${ }^{11}$ showed an interesting access through the upper wall of the nasal cavity and obstruction of the sinus ostium with cyanoacrylate, without violation of the maxillary sinus cavity in a model for studying ciliary movement in acute rhinosinusitis. Marks ${ }^{12}$ developed a model of rhinosinusitis by inoculating a foreign body in the nasal cavity along with a pathogen (S. pneumoniae). He considered this model feasible for the study of sinusitis, despite a loss of almost $46 \%$ of the original sample due to premature death or sacrifice (signs of systemic disease). To test a model of chronic rhinosinusitis, Kara et al. ${ }^{13}$ compared a group of rabbits which absorbable gelatin sponge was inserted between the endoturbinals and the ostium of the sinus and group packed with a sponge soaked in a solution containing Staphylococcus. In the group in which the ostium was occluded with sterile sponge, no evidence of sinusitis was seen. Seeking to create a chronic model consistent with rhinosinusitis with an access through the nasal cavity similar to Hinni's description, we were not effective in generating homogeneous findings consistent with CRS with the access without bacterial inoculation, as well as Kara's results in this group.

It is noteworthy that this is the first long-term model in sinusitis produced in Brazil e there were no losses, enhancing that the present model can be feasible and reproducible for future studies.

The present study was designed primarily to induce inflammatory changes in the sinus mucosa for histopathological observation; clearly, it was not the intention of the authors to the study the bacteriology of CRS. While the nature of acute sinusitis is infectious ${ }^{3}$, the disease is thought to result from a multifactorial inflammatory process, in which bacteria have an undefined role. Rabbits have a significant incidence of natural infection of paranasal sinuses, and Pasteurella multicida is the most common pathogen in laboratory rabbits. The high prevalence of bacterial growth by the end of the experiment, despite initial treatment for pausterelose, can be due to cross contamination, since animals were housed near other rabbits during the three months of followup. Moreover, it is interesting to note that the bacterial growth observed on the control side in most cases was not enough to cause chronic histological changes in these sinuses. On the other hand, in animals with negative cultures and histopathological findings compatible with CRS, it is not possible to exclude the role of bacteria in this pathogenesis (as biofilms, for example, which were 
not investigated in this model). This is a potential limitation of our study: since we are dealing with a chronic model, perhaps biofilms, rather than bacterial growth, are implicated in the pathophysiology of CRS. Nevertheless, further studies investigating the role of biofilms can be based on the current model, which has proven successful in inducing CRS. Furthermore, this model was also limited by the impossibility of reverting the chronic condition produced. However, it represents a consistent model for the generation of typically chronic cellular material, through which our knowledge of the intracellular and intercellular relationships can be advanced.

\section{Conclusions}

It is possible to induce a model of chronic sinusitis among rabbits with transmaxillary sinus occlusion without bacterial inoculation. On the other hand, the approach through the roof of the nasal cavity to occlude the middle meatus did not produce consistent results. This model can be replicated for future cellular studies.

\section{References}

1. Schlosser RJ, Spotnitz WD, Rodeheaver G, Scheld WM, Iezzoni J, Gross CW. Effects of fibrin sealant-containing antibiotics in a rabbit model of chronic sinusitis. Am J Rhinol. 2000;14(4), 233-40.

2. Khalid AN, Hunt J, Perloff JR, Kennedy DW. The Role of Bone in Chronic Rhinosinusitis. Laryngoscope. 2002;112(11), 1951-7.

3. Diretrizes Brasileiras de Rinossinusites. Rev Bras Otorrinolaringol. 2008;74 $\operatorname{Supl}(2): 6-59$.

4. Kumlien J, Schiratzki H. Blood flow in the rabbit sinus mucosa during experimentally induced chronic sinusitis. Measurement with a diffusible and with a non-diffusible tracer. Acta Otolaryngol. 1985;99(5-6):630-6.

5. Hilding A. Experimental sinus surgery: effects of operative windows on normal sinuses. Ann Otol Rhinol Laryngol. 1941;50:379-92.

6. Johansson P, Kumlien J, Söderlund K, Hultman E. Experimental acute sinusitis in rabbits. Energy metabolism in sinus mucosa and secretion. Acta Otolaryngol. 1988;106(5-6):460-7.

7. Westrin KM, Norlander T, Stierna P, Carlsöö B, Nord CE. Experimental maxillary sinusitis induced by Bacteroides fragilis. A bacteriological and histological study in rabbits. Acta Otolaryngol. 1992;112(1):107-14.

8. Fukami M, Norlander T, Stierna P, Westrin KM, Carlsöö B, Nord CE. Mucosal pathology of the nose and sinuses: a study in experimental maxillary sinusitis in rabbits induced by Streptococcus Pneumoniae, Bacteroides Fragilis, and Staphylococcus Aureus. Am J Rhinol. 1993;7(3):125-32.
9. Bolger WE, Leonard D, Dick EJ Jr, Stierna P. Gram negative sinusitis: a bacteriologic and histologic study in rabbits. Am J Rhinol. 1997;11(1):15-25.

10. Costa HO, Ruschi e Luchi GE, Augusto AG, Castro M, de Souza FC. Comparative study of several sinusitis experimental modelling techniques in rabbits. Braz J Otorhinolaryngol. 2007;73(5):627-31.

11. Hinni ML, McCaffrey TV, Kasperbauer JL. Early mucosal changes in experimental sinusitis. Otolaryngol Head Neck Surg. 1992;107(4): $537-48$.

12. Marks SC. Acute sinusitis in the rabbit: a new rhinogenic model. Laryngoscope. 1997;107(12 Pt 1):1579-85.

13. Kara CO, Cetin CB, Colakoğlu N, Sengül M, Pakdemirli E. Experimentally induced rhinosinusitis in rabbits. J Otolaryngol. 2002;31(5):294-8

\section{Acknowledgements}

All the staff at the Unit of Animal Experimentation who contributed to the implementation of the study, headed by Marta Cioato. Mario Wagner for the statistical counselor and Renata Rocha Kieling for helping in manuscript preparation in English language.

\section{Correspondence:}

Raphaella de Oliveira Migliavacca

Rua Ramiro Barcellos, 910/403

90035-001 Porto Alegre - RS Brasil

Tel./Fax: (55 51)3222-5886

raphaellamigliavacca@gmail.com

Received: Dec 19, 2013

Review: Feb 18, 2014

Accepted: March 20, 2014

Conflict of interest: none

Financial source: Hospital Research and Events Incentive Fund (FIPE/ HCPA)

${ }^{1}$ Research performed at Experimental Animal Unit, Porto Alegre Clinics Hospital, Porto Alegre-RS, Brazil. Part of Master degree thesis, Postgraduate Program in Medicine: Surgical Sciences, Rio Grande do Sul Federal University (UFRGS). Tutor: Sady Selaimen da Costa. 